\title{
Proto-planetary disks with CARMA: sub-arsecond observations at millimeter wavelengths
}

\author{
Isella Andrea ${ }^{1}$, John M. Carpenter ${ }^{1}$, Laura Perez ${ }^{1}$ and \\ Anneila I. Sargent ${ }^{1}$ \\ ${ }^{1}$ Department of Astronomy, California Institute of Technology, MC 249-17, Pasadena, CA \\ 91125. email:isella@astro.caltech.edu
}

Using the Combined Array for Research in Millimeter-wave Astronomy (CARMA) we observed several proto-planetary disks in the dust continuum emission at 1.3 and $2.8 \mathrm{~mm}$ (Isella et al. 2009a, 2009b). The observations have angular resolution between 0.15 and 0.7 arcsecond, corresponding to spatial scales spanning from about the orbit of Saturn up to about the orbital radius of Pluto. The observed disks are characterized by a variety of radial profiles for the dust density. We observe inner disk clearing as well as smooth density profiles, suggesting that disks may form, or evolve, in different ways. Despite that, we find that the characteristic disk radius is correlated with the stellar age increasing from $20 \mathrm{AU}$ to $100 \mathrm{AU}$ over about $5 \mathrm{Myr}$. Interpreting our results in terms of the temporal evolution of a viscous $\alpha$-disk, we estimate that (i) at the beginning of the disk evolution about $60 \%$ of the circumstellar material was located inside radii of $25-40$ AU, (ii) that disks formed with masses from 0.05 to 0.4 solar masses and (iii) that the viscous timescale at the disk initial radius is about 0.1-0.3 Myr. Viscous disk models tightly link the surface density $\Sigma(R)$ with the radial profile of the disk viscosity $\nu(R) \propto R^{\gamma}$. We find values of $\gamma$ ranging from -0.8 to 0.8 , suggesting that the viscosity dependence on the orbital radius can be very different in the observed disks. We demonstrate that the similarity solution for the surface density for $\gamma<0$ can explain the properties of some "transitional" disks without requiring discontinuities in the disk surface density. In the case of LkCa 15, a smooth distribution of material from few stellar radii to about 240 AU can produce both the observed SED and the spatially resolved continuum emission at millimeter wavelengths. For two sources, RY Tau and DG Tau, we observed the dust emission with a resolution as high as 0.15 arcsecond, which corresponds to a spatial scale of $20 \mathrm{AU}$ at the distance of the two stars. The achieved angular resolution is a factor 2 higher than any existing observation of circumstellar disks at the same wavelengths and enable us to investigate the disk structure with unprecedent details. In particular, we present a first attempt to derive the radial profile of the slope of the dust opacity $\beta$. We find mean values of $\beta$ of 0.5 and 0.7 for DG Tau and RY Tau respectively and we exclude that $\beta$ may vary by more than \pm 0.4 between 20 -70 AU. This implies that the circumstellar dust has a maximum grain size between $10 \mu \mathrm{m}$ and few centimeters.

\section{References}

Isella, A., Carpenter, J. M., \& Sargent, A. I. 2009a, ApJ 701, 260

Isella, A., Carpenter, J. M., \& Sargent, A. I. 2009b, in preparation. 